\title{
A INFLUÊNCIA DO ESTOQUE DE CAPITAL HUMANO SOBRE A PRODUTIVIDADE INDIVIDUAL: UMA ANÁLISE PARA A AGROPECUÁRIA E INDÚSTRIA EXTRATIVA
}

\section{THE INFLUENCE OF THE HUMAN CAPITAL STOCK ON INDIVIDUAL PRODUCTIVITY: AN ANALYSIS FOR AGRICULTURE AND EXTRACTIVE INDUSTRY}

\section{LA INFLUENCIA DEL STOCK DE CAPITAL HUMANO EN LA PRODUCTIVIDAD INDIVIDUAL: UN ANÁLISIS PARA LA INDUSTRIA AGRÍCOLA Y EXTRACTIVA}

\author{
Tatiane Salete Mattei ${ }^{1}$ \\ https://orcid.org/0000-0003-1652-0695 \\ Fernanda Mendes Bezerra ${ }^{2}$ \\ https://orcid.org/0000-0002-3307-0107
}

Submissão: 06/11/2020 / Aceito: 24/02/2021 / Publicado: 31/03/2021.

\begin{abstract}
Resumo
Partindo do pressuposto de que é possível estimar o salário de uma pessoa a partir das características pessoais, econômicas e locais, este trabalho objetiva verificar quais são as implicações do estoque de capital humano sobre a produtividade individual. Para alcançar ao objetivo, foram coletados dados da RAIS, referente aos trabalhadores da agropecuária e da indústria extrativa das 26 capitais dos estados brasileiros e da capital do Distrito Federal e, estimada uma Equação Minceriana com foco na teoria do capital humano. Os resultados mostraram que, ao investir em capital humano, os trabalhadores da agropecuária e da indústria extrativa se tornam mais produtivos. Verificou-se que um trabalhador com ensino superior completo aufere salário 3,3 vezes maior do que um trabalhador com ensino superior incompleto e, um trabalhador com pós-graduação ganha 1,6 vezes mais do que um trabalhador com o ensino superior completo. Ainda, um trabalhador com pós-graduação ganha 19 vezes mais que um trabalhador analfabeto. Verificou-se, também, que a qualidade da educação contribui de forma positiva para os trabalhadores se tornarem mais produtivos, os homens auferem salários maiores que as mulheres e os trabalhadores não são compensados com salários maiores se as condições do trabalho e da localidade são ruins.
\end{abstract}

Palavras-chave: Salário; Produtividade; Capital Humano.

\begin{abstract}
Based on the assumption that it is possible to estimate a person's salary based on personal, economic and local characteristics, this study aims to verify the implications of the stock of human capital on individual productivity. In order to reach the objective, RAIS data were collected, referring to agricultural and extractive workers in the 26 capitals of the Brazilian states and the capital of the Federal District, and an estimated Mincerian Equation focusing on the theory of human capital. The results showed that when investing in human capital, agricultural and extractive industry workers become more productive. It was found that a worker with complete higher education earns a salary 3.3 times higher than a worker with incomplete higher education and a worker with graduate

\footnotetext{
${ }^{1}$ Doutoranda no Programa de Pós-Graduação em Desenvolvimento Regional e Agronegócio pelo Unioeste, Campus Toledo- PR, bolsita Capes. Email: tati_mattei@ hotmail.com.

${ }^{2}$ Doutora em Economia pela Universidade Federal de Pernambuco- PE, Professora Adjunto da Universidade Estadual do Oeste do Paraná Campus Francisco Beltrão- PR. E-mail: ferpompeia@gmail.com.
} 
education earns 1.6 times more than a worker with complete higher education. Still, a graduate worker earns 19 times more than an illiterate worker. It was also found that the quality of education contributes positively to workers becoming more productive, men earn higher wages than women and workers are not compensated with higher wages if the conditions of work and location are poor.

Keywords: Salary; Productivity; Human capital.

\section{Resumen}

Partiendo del supuesto de que es posible estimar el salario de una persona en función de las características personales, económicas y locales, este trabajo tiene como objetivo verificar cuáles son las implicaciones del stock de capital humano en la productividad individual. Para lograr el objetivo, se recolectaron datos RAIS, referidos a trabajadores agrícolas y extractivos en las 26 capitales de los estados brasileños y la capital del Distrito Federal, y se estimó una Ecuación Minceriana con enfoque en la teoría del capital humano. Los resultados mostraron que, al invertir en capital humano, los trabajadores de la industria agrícola y extractiva se vuelven más productivos. Se encontró que un trabajador con educación superior completa gana un salario 3.3 veces mayor que un trabajador con educación superior incompleta y, un trabajador con educación de posgrado gana 1.6 veces más que un trabajador con educación superior completa. Aún así, un trabajador graduado gana 19 veces más que un trabajador analfabeto. También se encontró que la calidad de la educación contribuye positivamente a que los trabajadores se vuelvan más productivos, los hombres ganan salarios más altos que las mujeres y los trabajadores no son compensados con salarios más altos si las condiciones de trabajo y la ubicación son deficientes.
\end{abstract}

Palabras llave: Salario; Productividad; Capital humano.

\title{
INTRODUÇÃO
}

O Brasil apresenta diferencial de níveis educacionais elevados, impactando em grandes desigualdades na distribuição de renda da população, refletindo, também, em uma significativa incidência de pobreza (RAMOS; VIEIRA, 2001).

Os níveis de renda de uma sociedade são afetados e também afetam o nível de educação, saúde, nutrição, mortalidade, violência e várias outras características. Existe uma forte correlação entre educação e renda para, praticamente, todos os países do mundo. Assim, ao melhorar os níveis educacionais dos mais pobres, os níveis de renda também melhoram. (MEDEIROS; SOUZA; CASTRO, 2015; NAZARENO, 2016).

Segundo a teoria do capital humano, a educação e a experiência da população são responsáveis pelos diferenciais de produtividade, afetando diretamente os salários dos trabalhadores. A qualidade da educação e os transbordamentos da educação ocorridos muito em virtude das aglomerações, também tem papel relevante. Quanto maior a qualidade do capital humano, mais produtivo ele será (SCHULTZ, 1961, 1973). 
Além da teoria do capital humano e suas externalidades, outras teorias tentam explicar os diferenciais de produtividade e, em consequência, os diferenciais salariais no mercado de trabalho. A teoria dos diferenciais compensatórios atribui os diferenciais produtivos a algumas características das localidades onde os trabalhadores residem, como custos de vida e amenidades dos locais como: poluição, congestionamento, opções de lazer, entre outros (FERNANDES, 2002). Já a teoria da discriminação aponta que quando os trabalhadores com as mesmas capacidades produtivas, mas pertencentes a distintos grupos populacionais, ganham salários diferentes, ocorre a discriminação (FERNANDES, 2015).

Existe, ainda, a teoria da segmentação, na qual o indivíduo é remunerado de acordo com o setor ou ocupação em que está inserido no mercado de trabalho (SATEL, 2011). Diante dessa teoria, o setor agropecuário e a indústria extrativa foram escolhidos como escopo de análise pela sua importância para a economia.

O setor agropecuário respondeu por cerca de $21 \%$ do PIB brasileiro de 2018 e a indústria extrativa por cerca de 2,9\% (CEPEA, 2018, CNI, 2020). O setor agropecuário é visto como uma atividade altamente moderna, que exerce papel importante na dinâmica econômica, seja por assumir papel anticíclico pois, historicamente, tem apresentado altos volumes de exportações, mesmo em períodos de crise, resultando em saldos comerciais superavitários e crescentes, principalmente de 1998 a 2013, seja na geração de emprego e renda e no controle do êxodo rural (GARCIA, 2014; SANTOS et al., 2016).

$\mathrm{Na}$ indústria extrativa, a permanência na localidade do empreendimento, de grande parte da riqueza gerada pela atividade e a criação de empregos são fatores importantes para o desenvolvimento. Além disso, o setor é alvo de muitos estudos em virtude dos benefícios, mas, também, do custo que o setor pode causar para os ecossistemas e economias locais (LIMA, 2007).

Com foco na teoria do capital humano, surge, portanto, a problemática da pesquisa: Quais são as implicações do estoque de capital humano sobre a produtividade individual dos trabalhadores da agropecuária e indústria extrativa nas capitais brasileiras?

Assim, o objetivo geral do trabalho é estimar a influência e o grau do impacto do estoque de capital humano e suas externalidades sobre a produtividade individual dos trabalhadores da agropecuária e indústria extrativa das 26 capitais dos estados brasileiros e da capital do Distrito Federal. Além da teoria do capital humano e suas externalidades, o trabalho busca verificar a influência de outras variáveis, como discriminação e diferenciais compensatórios, na definição da produtividade individual e, consequentemente, dos salários. 
O recorte espacial escolhido se deu, em grande parte, pela disponibilidade de informações. Ademais, e apesar do reconhecimento de que a atividade primária, em regiões mais urbanizadas, não tem uma importância econômica elevada, pois os setores secundários e terciários acabam se sobressaindo, é notável que, nessas regiões, esse setor existe e essa atividade é bastante diversificada e importante para atender a alta demanda por alimentos. Com relação ao setor industrial, este ainda é pouco estudado (NUNES; MORAES; ROSSONI, 2020). Além disso, segundo Costa et al. (2013), a urbanização possibilita economias de escala na distribuição dos produtos primários e uma maior facilidade em relação a mão de obra.

Para alcançar os objetivos, será estimada uma equação Minceriana de rendimentos. Os dados utilizados na pesquisa são, sobretudo, provenientes da RAIS (Relação Anual de Informações Sociais), do ano de 2015, desenvolvido pelo Ministério do Trabalho.

O presente trabalho se diferencia dos demais por considerar muitas observações, analisar os dados no âmbito do indivíduo, além de incluir na equação minceriana variáveis proxy para a qualidade da educação e uma variável com o número de vagas universitárias por 1.000 habitantes como proxy para o acesso ao ensino. O trabalho considera importante não só o processo de ampliação do acesso à educação, que levou ao aumento da escolarização da população a partir dos anos 2000, mas a importância de uma educação de qualidade.

O estudo da determinação da produtividade, a partir da teoria do capital humano, se torna relevante, tanto para os formuladores de políticas públicas quanto para os trabalhadores brasileiros. Ao verificar que o capital humano contribui para o aumento da renda das famílias, levando a um maior consumo e, em consequência, a uma maior qualidade de vida, pode instigar os formuladores de políticas públicas a ampliar os gastos na área da educação, e fazê-los com maior responsabilidade e eficiência, a fim de reduzir as desigualdades de renda no país. Para os trabalhadores, pode ser um incentivo ao estudo e especialização visando a mobilidade social. Esse trabalho se divide em cinco seções, contando com essa introdução. Na segunda seção serão discutidas as teorias que embasam as escolhas das variáveis do modelo econométrico. A metodologia é apresentada na seção 3 e os resultados e discussões na seção 4. Por fim, são tecidas algumas considerações finais na quinta e última seção. 


\section{REFERENCIAL TEÓRICO}

Existe uma série de fatores que podem levar à existência de diferenças de salário entre os indivíduos e regiões e, portanto, afetar o nível da desigualdade de rendimento.

A teoria clássica aponta que, em mercados de trabalhos competitivos, sempre que houver excesso de oferta de trabalho, ou podemos dizer, desemprego, a concorrência por emprego levará a reduções de salários (LOPES; VASCONCELLOS, 2008).

Para Fontes, Simões e Oliveira (2006), as diferenças de salários entre regiões, dentro dos países, tendem a persistir ao longo do tempo e apresentam forte correlação com a densidade do tecido econômico local, ou seja, com as aglomerações.

Existem vantagens que as firmas obtêm com a concentração das atividades produtivas, como os encadeamentos a jusante e a montante, a presença de serviços complementares e infraestrutura básica como água, energia e infraestrutura logística. As empresas pagam salários nominais urbanos maiores para os funcionários, pois os ganhos de produtividade das firmas são repassados em algum grau para os trabalhadores. Porém, com esse processo, existem, também, alguns prejuízos para a população, como o declínio do bem-estar, e algumas amenidades ruins, como poluição, congestionamentos, além do preço do solo urbano (GALINARI et al., 2007).

As diferenças explicadas pela teoria dos diferenciais compensatórios estão associadas à heterogeneidade das localidades onde os trabalhadores residem, como custos de vida e amenidades dos locais. Os salários também podem ser desiguais em decorrência das diversas dotações de capital humano, expresso pela diferença na obtenção de educação e experiência dos indivíduos. Os diferenciais causados pela discriminação acontecem quando o mercado pode estar remunerando distintamente trabalhadores igualmente produtivos com base em atributos não produtivos, como cor e sexo, por exemplo. Além disso, um trabalhador pode ser remunerado de acordo com o setor ou ocupação em que está inserido no mercado de trabalho.

\section{Teoria do Capital Humano}

Capital humano pode ser definido como a capacidade produtiva que um indivíduo possui ou pode adquirir devido à acumulação de conhecimentos, utilizados na produção de riqueza. As atividades que aumentam a produtividade de um indivíduo são: educação formal, conhecimento, 
experiências, alimentação, saúde, ambiente familiar e cultural (VIANA; LIMA, 2010; RAIHER, 2017).

A teoria do capital humano ajuda a explicar as diferenças nos rendimentos ao longo do tempo e entre áreas mas, também, entre as pessoas e as famílias. Famílias mais ricas tendem a investir mais em capital humano em detrimento às famílias pobres. $\mathrm{O}$ fato de existirem dificuldades de financiamentos em educação contribui para isso (BECKER, 1962).

O capital humano melhora bem-estar das pessoas, além de ampliar o leque de escolhas disponíveis. O investimento em capital humano faz subir os salários reais por trabalhador, pois aumenta sua produtividade. Pessoas com maior educação e formação recebem ganhos quase sempre bem acima da média. Portanto, diferenciais de salários são diretamente proporcionais à educação. Há uma motivação para investir em educação pelo fato de que, ao aumentar os anos de estudo, as pessoas podem aumentar seu nível de renda (SCHULTZ, 1961, 1973; BECKER, 1994).

Segundo Schultz (1987), o capital humano contribui também para a capacidade empreendedora das pessoas, tanto nas atividades ligadas à agricultura, quanto à indústria e produção doméstica. Tal capacidade proporciona melhores oportunidades de emprego, levando a um maior consumo futuro.

Os acréscimos nos salários das pessoas são cada vez menores para níveis mais altos de escolaridade, assim como os rendimentos marginais decrescentes existentes no mercado. Sendo assim, existem rendimentos decrescentes com a escolaridade adicional e investimentos decrescentes também de acordo com a idade e experiência (BECKER, 1962).

Hanushek e Kimko (2000) incluíram a qualidade do capital humano como fator importante para explicar a superioridade de alguns países em relação a taxa de crescimento econômico. Apesar da incerteza da medição da qualidade do capital humano, testaram, empiricamente e, apontaram que a qualidade da força de trabalho está diretamente relacionada à produtividade dos trabalhadores e ao crescimento econômico.

Moretti (2004) cita que a concentração de capital humano influencia a produtividade dos trabalhadores, pois um aumento na proporção de trabalhadores mais escolarizados em uma cidade eleva o salário de todos os trabalhadores dessa cidade, até mesmo dos sem instrução devido ao efeito spillover, ou seja, há um transbordamento de conhecimento, levando a um aumento de produtividade de todos.

Para Romer (1986), o investimento em conhecimento gera um tipo de externalidade natural em que a criação de novos conhecimentos por uma empresa, por exemplo, tem um efeito externo 
positivo nas possibilidades de produção de outras empresas porque o conhecimento não pode ser perfeitamente patenteado ou mantido em segredo.

A magnitude das externalidades do capital humano está enraizada em problemas metodológicos de identificação. Apesar dos desafios, evidências empíricas sugerem que as externalidades de capital humano são determinantes da produtividade e dos salários e, portanto, constituem um importante enigma do desenvolvimento regional (HEUERMANN, 2009).

\section{Teoria dos Diferenciais Compensatórios}

Os salários podem ser afetados por uma multiplicidade de outros fatores, como os custos de alugueis, o preço da terra e a compensação, devido às amenidades existentes, como o clima, opções de lazer e cultura (HEUERMANN; HALFDANARSON; SUEDEKUM, 2010),

A teoria dos diferenciais compensatórios explica os diferenciais salariais entre postos de trabalhos segundo as características onerosas, como risco de acidente e doenças. Para os diferenciais regionais de salários, a teoria é explicada em virtude das condições de vida de determinada região, como crime, congestionamentos, poluição, entre outros. Para explicar diferenciais de salários relacionados ao tempo de permanência no emprego, há a fixação de turnos, risco de desemprego, flexibilidade da jornada de trabalho, entre outros (FERNANDES, 2002).

Galinari et al. (2007) explicam que a força de trabalho não se concentra, em sua totalidade, nos grandes centros, onde são ofertados os maiores salários, devido aos diferenciais de estilos de vida e das oportunidades que as cidades podem oferecer. Nas grandes aglomerações, existem mais opções de conforto, lazer e serviços, como restaurantes étnicos, cinemas, serviços de entrega e teatros, sendo estes considerados fatores aglomerativos. Por outro lado, perdem para as cidades menores em termos de amenidades ambientais, como o tráfego, ruídos, níveis de criminalidade e de poluição. Assim, conclui-se que os trabalhadores, sob as mesmas condições de educação e experiência, aceitam salários reais relativamente diferentes porque agem racionalmente em função de compensações que recebem em termos de qualidade de vida.

Os diferenciais positivos, ou seja, salários mais altos, acompanham as características ruins de um emprego ou localidade, enquanto diferenciais negativos, salários mais baixos, estão relacionados com características boas (EHRENBERG; SMITH, 2000). 


\section{Teoria da Discriminação}

A definição ou hipótese amplamente aceita é de que objetos idênticos têm o mesmo preço. Considerando a força de trabalho como mercadoria, dois indivíduos com a mesma produtividade devem ter o mesmo salário. Quando isso não acontece, ou seja, quando trabalhadores pertencentes a distintos grupos populacionais, mas com as mesmas capacidades produtivas ganham salários diferentes, ocorre a discriminação (FERNANDES, 2015).

A teoria da discriminação analisa a situação desfavorável que alguns grupos da população estão sujeitos devido às características não econômicas, como raça, gênero, religião, idade, entre outros (ROCHA; PERO, 2007; CHAVES, 2011).

Silva e Kassouf (2000) encontram a existência de uma desigualdade substancial nos rendimentos entre homens e mulheres, a qual se chama discriminação salarial por gênero. Essa discriminação existe quando o mercado de trabalho remunera distintamente trabalhadores igualmente produtivos com base em atributo não produtivo - nesse caso, o sexo.

Cacciamali e Hirata (2005), a respeito da discriminação por raça e gênero, sugerem que no Brasil a herança escravocrata e a herança patriarcal implicam elevado grau de desigualdade de rendimentos no mercado de trabalho.

Apesar de não ser objetivo do presente trabalho analisar a fundo a discriminação por gênero e raça, será incluído no modelo uma variável controle para o sexo e raça dos trabalhadores para verificar quem aufere mais renda na agropecuária e indústria extrativa das capitais brasileiras.

\section{Teoria da Segmentação}

A partir das últimas décadas do século XX, se desenvolveu com mais corpo uma teoria alternativa que visa explicar a determinação de salários e a mobilidade ocupacional, chamada de teoria da segmentação com base em análises do mercado de trabalho norte-americano (SILVA, 2006).

Segundo a teoria da segmentação, o indivíduo é remunerado de acordo com o setor ou ocupação em que está inserido no mercado de trabalho (SATEL, 2011). A educação, nessa teoria, tem papel de dar acesso a certos segmentos, ou seja, o nível educacional fornece um sinal que determina a que mercado de trabalho a pessoa vai ter acesso (SILVA, 2006). 
Silva (2006) argumenta que, na teoria da segmentação, os salários têm maior ligação com os tipos de empregos a que o trabalhador tem acesso e com a forma de organização do trabalho nas indústrias ou nos escritórios do que com as características do trabalhador.

Fernandes (2002) propôs uma definição mais técnica, definiu segmentação como sendo a situação em que trabalhadores com as mesmas preferências e produtividade obtêm níveis de utilidade distintos por ocuparem postos de trabalho diferentes. Além disso, afirmou que os mecanismos de mercado falhariam na equalização dessas diferenças.

Fernandes (2002) ainda aponta como possíveis causas da segmentação a existência de leis trabalhistas e sindicatos. As leis trabalhistas constituem-se, no argumento mais utilizado, como a intervenção de governos que podem criar segmentação no mercado de trabalho. Para ele, a legislação de salários, ou sindicatos, pode impor remuneração mais elevada do que o ordenado competitivo do mercado e, assim, gerar racionamento, desemprego e dualismo salarial.

\section{METODOLOGIA}

Partindo do pressuposto de que é possível estimar os salários de uma pessoa a partir de características pessoais, econômicas e locais, este trabalho investiga a relação entre capital humano e suas externalidades, além de outros fatores, como aglomerações, amenidades, discriminação, a partir de estimações de equações mincerianas.

Este estudo tem abordagem quantitativa, a qual, com relação ao seu objetivo, é caracterizada como descritiva. São utilizados os microdados da Relação Anual de Informações Sociais (RAIS), ou seja, dados do indivíduo como unidade de observação para o ano de 2015. A RAIS considera apenas o mercado formal. As unidades geográficas de análise são as 26 capitais dos estados brasileiros e a capital do Distrito Federal, uma vez que representam grande aglomerado de pessoas e indústrias nessas localidades, contemplando a teoria da aglomeração, além de maior disponibilidade de informações. Ademais, o setor agropecuário tem importância econômica e é pouco estudado fora das grandes regiões com tradição agrícola.

A população do estudo compreende todos os trabalhadores formais da agricultura, pecuária, produção florestal, pesca, aquicultura e indústria extrativa (será simplesmente chamada de agropecuária e indústria extrativa) registrados na RAIS. Essa delimitação de setor contempla a teoria da segmentação abordada no referencial. Para ter melhor desempenho no software de análise, foi escolhida uma amostra com base em alguns filtros (horas contratuais entre 40 e 44, remuneração 
declarada, informações válida para raça e com idade entre 18 e 70 anos), com isso obteve-se uma amostra de 16.986 trabalhadores.

Com a Equação Minceriana é possível estimar os salários de um trabalhador por meio de uma equação com o logaritmo do salário/horas de trabalho como variável dependente e um conjunto de atributos produtivos como variáveis independentes. Também é utilizada para medir o diferencial de salários entre diferentes grupos, diferentes ocupações, ou para diferenças de gênero (CARVALHO; NERI; SILVA, 2006; TATEI, 2011).

A forma inicial da Equação Minceriana é apresentada na Equação 1:

$$
L n \omega_{\mathrm{i}}=\beta_{0}+\beta_{1} E_{1}+\beta_{2} T_{\mathrm{i}}+\beta_{3} T_{\mathrm{i}}^{2}+X_{\mathrm{i}}+\varepsilon_{\mathrm{i}}
$$

$L n \omega_{\mathrm{i}}$ representa o logaritmo natural do salário em determinado período, $E_{1}$ representa os anos de estudo de um indivíduo, $T_{\mathrm{i}}$ representa a experiência do indivíduo no mercado de trabalho, $T_{\mathrm{i}}{ }^{2}$ é uma variável utilizada para captar o retorno de longo prazo da experiência. $X_{\mathrm{i}}$ é um vetor das características produtivas e não produtivas que também impactam sobre o rendimento do trabalhador e $\varepsilon_{\mathrm{i}}$ representa o erro ou o resíduo (FÁVERO; et al., 2009).

O método utilizado para estimar as equações mincerianas será o método dos mínimos quadrados ordinários (MQO). Segundo Rocha, Neto e Gomes (2016), o MQO possui a vantagem de estimar o modelo controlando para as características observáveis e não observáveis invariantes no tempo captadas pelo efeito fixo do modelo, mas que influenciam o salário dos trabalhadores. Para dar mais robustez e confiança à regressão, será testada a significância das variáveis e do modelo, os pressupostos como: normalidade, homocedasticidade e multicolinearidade, bem como, o coeficiente de determinação da regressão $\left(\mathrm{R}^{2}\right)$. Quanto maior for $\mathrm{o} \mathrm{R}^{2}$, ou seja, quanto mais próximo de 1 , melhor é a qualidade do ajustamento do modelo (GUJARATI, PORTER, 2012).

A variável dependente $L n \omega_{\mathrm{i}}$ será representada pelo logaritmo da razão entre o salário e a jornada de trabalho em horas. Essa forma funcional é chamada de semilog e é bem flexível, pois se pode incluir diversas outras variáveis. A equação minceriana deste trabalho incluirá variáveis que captarão os efeitos do capital humano (escolaridade, experiência e experiência ${ }^{2}$ ). A escolaridade será representada por Dummies, para trabalhador analfabeto, ensino fundamental completo, ensino médio completo e incompleto, ensino superior completo e incompleto, e pós-graduação. Será atribuído valor 1 se o trabalhador tem a escolaridade determinada e 0 se não. Como proxy da experiência será utilizado tempo no atual emprego e para experiência ao quadrado o tempo no atual emprego ao quadrado. Conforme foi utilizada por Mattei e Baço (2016, p. 875), a experiência ao quadrado será usada com a justificativa de captar os retornos marginais decrescentes, significando "quanto mais 
experiência um trabalhador possuir, maior será a sua remuneração, no entanto, chega um momento da vida do trabalhador que o retorno sobre a experiência vai crescendo a taxas decrescentes", o esperado é que essa variável apresente sinal positivo.

A equação incluirá também os efeitos das externalidades do capital humano - spillover (proporção de trabalhadores qualificados com no mínimo ensino superior completo), efeitos advindos das aglomerações (densidade demográfica e a taxa de desocupação), efeitos dos diferenciais compensatórios (tarifas médias de energia elétrica como proxy do custo de vida e número de leitos disponíveis em hoteis por 100.000 habitantes como proxy de amenidades boas ou bem estar), efeitos da discriminação (controles para sexo e raça). Como diferencial o trabalho incluirá uma variável que capta a influência do acesso à educação para a produtividade (vagas universitárias por mil habitantes), representando a função econômica de inserção da educação e, também, uma variável que capta a qualidade da educação (Índice Firjan de Desenvolvimento Municipal -IFDM-educação).

A forma funcional do modelo geral será estimada conforme Equação 2:

$$
\operatorname{LnW}_{i j}=\beta^{0}+\beta \operatorname{Exp}_{i j}+\beta \operatorname{Exp}_{i j}^{2}+\beta \text { Analf }_{i j}+\beta \text { Fund }_{i j}+\beta \text { MédioIncom }_{i j}+
$$

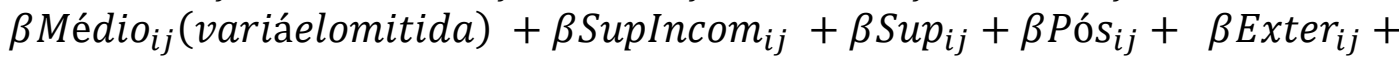
$\beta$ DensDem $_{i j}+\beta$ CMtrab $_{i j}+\beta$ Custovida $_{i j}+\beta$ Amenidades $_{i j}+\beta$ Negro $_{i j}+\beta$ Homem $_{i j}+$ $\beta$ Quali $_{i j}+\beta$ VagasUniv $_{i j}+\varepsilon_{i j}$

No Quadro 1 estão relacionadas as variáveis, uma descrição sucinta de cada variável e de onde foram coletadas.

\section{Quadro 1- Variáveis do estudo}

\begin{tabular}{|c|c|c|c|}
\hline Variáveis & Nome atribuído & Descrição & $\begin{array}{l}\text { Base de dados e ano } \\
\text { de referência }\end{array}$ \\
\hline \multicolumn{4}{|c|}{ VARIÁVEL DEPENDENTE } \\
\hline Ln W & $\begin{array}{l}\text { Logaritmo do salário por } \\
\text { hora }\end{array}$ & $\begin{array}{l}\text { Logaritmo da razão entre o } \\
\text { salário e a jornada de trabalho } \\
\text { em horas. }\end{array}$ & RAIS, 2015 \\
\hline \multicolumn{4}{|c|}{ INTERCEPTO DA REGRESSÃO } \\
\hline$\beta_{0}$ & Coeficiente angular & $\begin{array}{llll}\begin{array}{l}\text { Intercepto } \\
\text { constante. }\end{array} & \text { da } & \text { equação } & \text { ou } \\
\end{array}$ & - \\
\hline \multicolumn{4}{|c|}{ VARIÁVEIS INDEPENDENTES } \\
\hline \multicolumn{3}{|c|}{ CAPITAL HUMANO } & \\
\hline$\beta_{1} \operatorname{Exp}$ & Experiência & Tempo no atual emprego. & RAIS, 2015 \\
\hline$\beta_{2} \operatorname{Exp}^{2}$ & Experiência $^{2}$ & $\begin{array}{l}\text { Tempo no atual emprego ao } \\
\text { quadrado. }\end{array}$ & RAIS, 2015 \\
\hline$\beta_{3}$ Analf & Analfabeto & $\begin{array}{l}\text { Dummy com valor } 1 \text { para } \\
\text { trabalhadores analfabetos. }\end{array}$ & RAIS, 2015 \\
\hline$\beta_{4}$ Fund & Fundamental & $\begin{array}{l}\text { Dummy com valor } 1 \text { para } \\
\text { trabalhadores com } 1^{\text {a }} \text { etapa do } \\
\text { ensino fundamental completo, }\end{array}$ & RAIS, 2015 \\
\hline
\end{tabular}


Revista Grifos - Unochapecó

\begin{tabular}{|c|c|c|c|}
\hline & & $\begin{array}{l}2^{a} \text { etapa do ensino fundamental } \\
\text { completo e incompleto. }\end{array}$ & \\
\hline$\beta_{5}$ Médio Incom & Médio incompleto & $\begin{array}{l}\text { Dummy com valor } 1 \text { para } \\
\text { trabalhadores com ensino médio } \\
\text { incompleto. }\end{array}$ & $R A S, 2015$ \\
\hline$\beta_{6}$ Médio & Médio & $\begin{array}{l}\text { Dummy com valor 1 para } \\
\text { trabalhadores com ensino médio } \\
\text { completo. Essa foi a variável } \\
\text { omitida para fins de } \\
\text { comparação. }\end{array}$ & RAIS, 2015 \\
\hline$\beta_{7}$ Sup Incom & Superior incompleto & $\begin{array}{l}\begin{array}{l}\text { Dummy com valor } \\
\text { 1para } \\
\text { trabalhadores com } \\
\text { superior incompleto. }\end{array} \\
\text { ensino }\end{array}$ & RAIS, 2015 \\
\hline$\beta_{8}$ Sup & Superior & $\begin{array}{lrrr}\text { Dummy com valor } & 1 & \text { para } \\
\text { trabalhadores com } & \text { ensino } \\
\text { superior completo. } & & \\
\end{array}$ & RAIS, 2015 \\
\hline$\beta_{9}$ Pós & Pós-graduação & $\begin{array}{l}\text { Dummy com valor } 1 \text { para } \\
\text { trabalhadores com mestrado e } \\
\text { doutorado. }\end{array}$ & RAIS, 2015 \\
\hline EXTE & ALIDADES DO CAPITAL HI & MANO- SPILLOVER & \\
\hline$\beta_{10}$ Exter & $\begin{array}{l}\text { Externalidades do capital } \\
\text { humano }\end{array}$ & $\begin{array}{l}\text { Proporção de trabalhadores com } \\
\text { no mínimo superior completo } \\
\text { por setor de cada grande região } \\
\text { brasileira. }\end{array}$ & RAIS, 2015 \\
\hline & AGLOMERAÇÕE & & \\
\hline$\beta_{11}$ DensDem & Densidade Demográfica & $\begin{array}{l}\text { Relação entre a população e a } \\
\text { superfície do território. }\end{array}$ & IBGE, 2010. \\
\hline$\beta_{12} \mathrm{CM}$ trab & $\begin{array}{l}\text { Condição do mercado de } \\
\text { trabalho }\end{array}$ & Taxa de desocupação & $\begin{array}{l}\text { PNAD CONTÍNUA, } \\
2015 .\end{array}$ \\
\hline & DIFERENCIAIS COMPENS & ATÓRIOS & \\
\hline$\beta_{13}$ Custo Vida & Custo de Vida & $\begin{array}{l}\text { Valor médio da tarifa de energia } \\
\text { elétrica por Quilowatt }(\mathrm{KW}) \text { das } \\
\text { capitais. }\end{array}$ & $\begin{array}{lr}\text { Agência } & \text { Nacional de } \\
\text { Energia } & \text { Elétrica } \\
\text { (ANEEL), } 2015 .\end{array}$ \\
\hline$\beta_{14}$ Amenidades & Amenidades Locais & $\begin{array}{l}\text { Quantidade de leitos disponíveis } \\
\text { do setor hoteleiro por } 100.000 \\
\text { habitantes. }\end{array}$ & IBGE, 2016. \\
\hline & DISCRIMINAÇÃ & & \\
\hline$\beta_{15}$ Homem & $\begin{array}{l}\text { Trabalhadores do sexo } \\
\text { masculino }\end{array}$ & $\begin{array}{l}\text { Dummy com valor } 1 \text { se homem } \\
\text { e valor } 0 \text { se mulher. }\end{array}$ & RAIS, 2015 \\
\hline$\beta_{16}$ Negro & $\begin{array}{l}\text { Trabalhadores declarados } \\
\text { negros }\end{array}$ & $\begin{array}{l}\text { Dummy com valor } 1 \text { para } \\
\text { declarados negros, para os não } \\
\text { negros aplicado } 0 \text {. }\end{array}$ & RAIS, 2015 \\
\hline & QUALIDADE DA EDUC & $\mathrm{AÇ} \tilde{\mathrm{A} O}$ & \\
\hline$\beta_{23}$ Quali & Qualidade da educação & $\begin{array}{l}\text { Índice IFDM da dimensão } \\
\text { educação de cada capital. }\end{array}$ & $\begin{array}{l}\text { FIRJAN, } 2015 \text { ANO } \\
\text { BASE } 2013 .\end{array}$ \\
\hline & VAGAS UNIVERSITÁ & RIAS & \\
\hline$\beta_{24}$ Vagas Uni & Vagas Universitárias & $\begin{array}{l}\text { Vagas oferecidas em cada } \\
\text { município na graduação } \\
\text { presencial por mil habitantes. }\end{array}$ & $\begin{array}{lr}\text { Censo } & \text { ensino } \\
\text { superior, } & 2010 . \\
\text { Divulgado pelo } & \text { INEP. }\end{array}$ \\
\hline & ERRO & & \\
\hline$\varepsilon$ & Termo de erro da equação & $\begin{array}{l}\text { Representa as características } \\
\text { não observáveis dos } \\
\text { trabalhadores. }\end{array}$ & - \\
\hline
\end{tabular}

FONTE: Elaborado pelas autoras, 2020. 
A variável tempo de emprego é proxy para a experiência dos trabalhadores e, se espera que apresente sinal positivo, indicando que o tempo de experiência aumenta a produtividade, levando a maiores rendimentos. O tempo de emprego ao quadrado indica o que Becker (1962) afirma sobre rendimentos marginais decrescentes de acordo com o aumento da idade e da experiência, o sinal esperado é negativo. A respeito das variáveis acesso à educação e qualidade da educação, se espera que apresentem sinais positivos, influenciando positivamente para aumento de produtividade.

A variável externalidade indicará se há um transbordamento de conhecimento dos mais qualificados, levando a um aumento da produtividade de todos, o sinal esperado é positivo. Esperase que a variável densidade demográfica apresente sinal positivo, indicando que, nas aglomerações, as pessoas recebem salários maiores e a variável condição do mercado (taxa de desocupação) de trabalho apresente sinal negativo, indicando que, quando existe desemprego alto, a concorrência por vagas leva a salários menores.

Seguindo o que diz a teoria dos diferenciais compensatórios, se espera que o sinal da variável custo de vida seja positivo, indicando uma compensação para que os trabalhadores ganhem salários maiores. A respeito da variável amenidades locais, segundo Heuermann (2009), a oferta de camas de hoteis em uma região indica que essa localidade atrai pessoas, seja por motivo de lazer ou negócios, demonstrando ser um local agradável, que proporciona bem-estar social. Se espera que tenha sinal negativo, indicando a relação inversa de salários mais altos atrelados às características ruins de um emprego e os salários mais baixos relacionados às características boas.

No presente trabalho não será aprofundada a questão da discriminação salarial por sexo e raça no mercado de trabalho mas, serão incluídas variáveis dummies para tentar captar se existe tendência de salários maiores para alguns desses grupos. A dummy homem receberá valor 1 para os trabalhadores homens, caso contrário receberão valor 0 . A dummy negro receberá valor 1 para os trabalhadores declarados negros segundo informações da RAIS (categoria 4), para os trabalhadores declarados brancos, pardos, indígenas e amarelos será atribuído valor 0. Arraes e Mariano (2014), assim como Soares (2000), também utilizaram uma variável categórica para sexo em seus estudos.

\section{RESULTADOS}

Antes da discussão dos resultados econométricos, na Tabela 1 é apresentada uma análise descritiva das variáveis utilizadas no modelo e, na Tabela 2, uma análise da frequência das variáveis dummies. 
Tabela 1- Estatística descritiva das variáveis

\begin{tabular}{c|c|c|c|c}
\hline Variáveis (exceto dummies) & Média & $\begin{array}{c}\text { Desvio } \\
\text { Padrão }\end{array}$ & Mínimo & Máximo \\
\hline Valor remuneração (R\$) & $1.510,84$ & $2.684,88$ & 240,00 & $56.419,69$ \\
Horas trabalhadas na semana & 43,84 & 0,77 & 35 & 44 \\
Tempo emprego (meses) & 29,74 & 57,70 & 0 & 566,9 \\
Salário por hora & 35,28 & 66,89 & 5,45 & $1.410,49$ \\
IFDM educação de cada capital & 0,763 & 0,074 & 0,557 & 0,904 \\
Externalidade da educação de cada capital & 2,54 & 1,43 & 0,43 & 8,77 \\
Densidade demográfica de cada capital & $2.022,98$ & $2.630,75$ & 12,57 & $7.786,44$ \\
Taxa de desocupação (\%) de cada capital & 8,80 & 2,53 & 4,8 & 14,1 \\
Tarifa energia elétrica por KW de cada capital & 0,55 & 0,14 & 0,30 & 0,89 \\
Leitos setor hoteleiro por 100.000 habitantes de cada capital & 2,95 & 0,90 & 0,73 & 5,27 \\
Vagas universitárias por 1.000 habitantes de cada capital & 28,78 & 9,65 & 7,61 & 44,09 \\
\hline
\end{tabular}

Fonte: Elaborado pelas autoras com base em RAIS (2015), IBGE (2010), PNAD CONTÍNUA (2015), ANEEL (2015), IBGE (2016), FIRJAN (2015), INEP (2010).

O setor agropecuário e extrativo das capitais brasileiras gerou uma amostra de 16.986 trabalhadores que obtiveram uma remuneração média de $\mathrm{R} \$ 1.510,84$ e jornada de trabalho média de 43,84 horas semanais. O Brasil é um país muito heterogêneo e o cenário dentre as capitais segue a mesma linha, como se observa no alto desvio padrão da maioria das variáveis e na diferença entre máximo e mínimo.

\section{Tabela 2- Tabela de frequência variáveis dummies}

\begin{tabular}{c|c|c|c|c}
\hline \multirow{2}{*}{ Variável Dummy } & \multicolumn{2}{|c|}{$\mathbf{1}$} & \multicolumn{2}{c}{ 0 } \\
\cline { 2 - 5 } & Frequência & \% & Frequência & \% \\
\hline Sexo masculino & 13.664 & 80,44 & 3.322 & 19,56 \\
Declarados negros & 1.026 & 6,04 & 15.960 & 93,96 \\
Analfabeto & 4.580 & 26,96 & 12.406 & 73,04 \\
Fundamental (completo e incompleto) & 6.884 & 40,53 & 10.102 & 59,47 \\
Médio incompleto & 1.144 & 6,73 & 15.842 & 93,27 \\
Médio completo & 4.290 & 25,26 & 12.696 & 74,74 \\
Superior incompleto & 244 & 1,44 & 16.742 & 98,56 \\
Superior completo & 896 & 5,27 & 16.090 & 94,73 \\
Pós-graduação & 26 & 0,15 & 16.960 & 99,85 \\
\hline
\end{tabular}

Fonte: Elaborado pelas autoras com base em RAIS (2015).

Nota 1: os trabalhadores que possuem o atributo receberam 1, caso contrário 0.

A maioria dos trabalhadores da agropecuária e da indústria extrativa das capitais brasileiras é do sexo masculino e declarados não negros. Prevalecem trabalhadores com apenas o ensino fundamental, em segundo lugar analfabetos e, em terceiro, pessoas com o ensino médio completo. Chama a atenção que apenas 26 trabalhadores do setor agropecuário e extrativo, em todas as capitais, possui pós graduação. Esses dados mostram que o gargalo do setor é a educação, pois o grau de instrução dos produtores é muito relevante. Interfere na facilidade de obtenção e processamento de 
informações, bem como a maneira correta de utilizar essas informações para o processo produtivo. Um produtor com maior grau de instrução consegue gerir a propriedade de forma mais segura e assertiva (ARRAIS, PRAT, CAMBRAIA, 2019).

$\mathrm{Na}$ Tabela 3 são apresentados os resultados da equação minceriana. A significância do modelo testado através do teste $\mathrm{F}$ apresentou valor de $p$ menor que 0,05 , indicando que o modelo é confiável.

A significância das variáveis foi testada através do teste $T$, as variáveis externalidade, densidade demográfica e negro não foram significativas. As variáveis tempo de emprego e sexo foram significativas ao nível de confiança de $90 \%$. As demais variáveis foram significativas ao nível de $99 \%$ e erro de $1 \%$. O pressuposto da normalidade foi alcançado justificado pelo fato do número de observações do modelo ser alto. Os outros pressupostos da homocedasticidade e ausência de multicolinearidade foram satisfeitos. $\mathrm{O} \mathrm{R}^{2}$ do modelo foi 0,5060 , ou seja, 50,60\% da variação do logaritmo de salário por hora é explicado pelas variáveis do modelo. Segundo Fávero et al. (2009), o valor ideal indicado de $\mathrm{R}^{2}$ é estar acima de $30 \%$.

Tabela 3 -Resultados da Estimação da Equação Minceriana da Variável Dependente log. do salário/horas trabalhadas da Agropecuária e Indústria extrativa

\begin{tabular}{|c|c|}
\hline VARIÁVEL & COEFICIENTE \\
\hline Tempo emprego & $0.0003396 * * *$ \\
\hline Tempo emprego ${ }^{2}$ & $4.4700000^{*}$ \\
\hline Densidade demográfica & $(-2.1200000)$ \\
\hline Custo Vida & $-0.3245454^{*}$ \\
\hline Taxa desocupação & $-0.0101815^{*}$ \\
\hline IFDM educação & $0.4791649 *$ \\
\hline Vagas universitárias/1.000 & $-0.0022783^{*}$ \\
\hline Externalidades & $(0.0001568)$ \\
\hline Amenidades Locais & $0,0000487 *$ \\
\hline D Homem & $0.0174431 * * *$ \\
\hline D Negro & $(-0.0022441)$ \\
\hline D analfabeto & $-0.914643 *$ \\
\hline D fundamental & $-0.0351082 *$ \\
\hline D médio incompleto & $-0.0747023^{*}$ \\
\hline D superior incompleto & $0.3567388 *$ \\
\hline D superior & $1.551189^{*}$ \\
\hline D pós & $2.031216^{*}$ \\
\hline Constante & 3.055955 \\
\hline Número Observações & 16.986 \\
\hline $\mathrm{R}^{2}$ & 0,5060 \\
\hline
\end{tabular}

Fonte: Resultados da pesquisa, 2020.

Nota $1: *$ significante a $1 \%, * *$ significante a $5 \%, * * *$ significante a $10 \%$.

Nota 2: Entre parêntese não foi significativa.

Nota 3: Variável omitida: D médio. 
A variável tempo de emprego, proxy escolhida para experiência, se apresentou significativa e com sinal positivo, indicando que a experiência dos trabalhadores tem influência na determinação da remuneração dos indivíduos, apesar de ter um coeficiente baixo. Esse resultado está de acordo com a teoria do capital humano, principalmente sobre o capital humano específico que cita Becker (1962), aquele que se refere a treinamentos e experiências, como um determinante para aumento de produtividade.

A variável tempo de emprego ao quadrado apresentou sinal positivo, indicando que para esse setor há retornos marginais crescentes, ou seja, conforme o trabalhador vai adquirindo mais tempo de experiência no trabalho, sua remuneração vai aumentando a taxas crescentes, e não decrescentes conforme cita a literatura.

O valor da tarifa de energia elétrica, proxy para o custo de vida, apresentou sinal negativo, ao contrário do que se esperava, indicando que o custo de vida (amenidade ruim) não interfere para os trabalhadores receberem incrementos em sua remuneração, mas sim, interfere para reduzir a renda dos trabalhadores. Esse resultado pode indicar que essa variável não é uma proxy adequada. Em Rocha et al. (2014), foi utilizado como proxy do custo de vida o valor do aluguel mensal e essa variável se apresentou positiva, porém com valor bem reduzido. Não foi possível utilizar a mesma proxy no presente estudo por não ter as informações necessárias para todas as capitais.

A variável taxa de desocupação apresentou sinal negativo, indicando que, quando existe alto desemprego, os salários dos trabalhadores tendem a ser menores, devido à alta concorrência por vagas de emprego. Esse resultado está de acordo com a Teoria Clássica do mercado de trabalho que diz que quanto maior é a oferta de mão de obra menor será a remuneração oferecida pelo empregador.

A influência do desemprego no nível de rendimentos dos trabalhadores reforça a importância de políticas que atuam positivamente na saúde, educação, treinamento, ampliando possibilidades de acesso a bens e serviços. A qualificação é necessária, mas insuficiente, precisa-se também passagem das atividades tradicionais para aquelas de maior complexidade tecnológica (inserção mais dinâmica das economias nacionais no mercado mundial), consolidação de políticas adequadas de investimento, poupança e estabilidade das principais variáveis econômicas (RIVANEDEIRA, 2000).

Um dos diferenciais do presente trabalho é a inclusão da variável vagas universitárias por mil habitantes como proxy para o acesso à educação e inclusão da variável IFDM- educação como 
proxy da qualidade da educação. A variável vagas universitárias apresentou sinal negativo indicando que a disponibilidade de vagas influencia negativamente o salário dos trabalhadores.

A variável IFDM educação apresentou sinal positivo, indicando que a qualidade da educação contribui de forma positiva para os trabalhadores se tornarem mais produtivos. No aspecto da qualidade, este resultado está de acordo com a teoria dos diferenciais compensatórios em que Ciccone e Peri (2006) citam que os trabalhadores com o mesmo capital humano ganham salários diferentes em equilíbrio, pois as cidades possuem características diferentes que são relevantes para a utilidade dos trabalhadores como custo de habitação, qualidade das escolas públicas, taxas de impostos, grau de poluição do ar, criminalidade, clima, opções de lazer, entre outros.

A proxy escolhida para as amenidades locais foi: leitos de hoteis disponíveis por 100.000 habitantes porém, seu sinal positivo foi contra ao que se esperava, indicando, assim, que salários altos para os trabalhadores da agropecuária e indústria extrativa não estão atrelados a características boas ou ruins de um emprego, ou seja, os trabalhadores não são compensados com salários maiores se as condições de trabalho são ruins e, recebem salários menores se as condições são boas.

A variável homem apresentou sinal positivo, podendo-se concluir que os homens auferem salários maiores que as mulheres na agropecuária e indústria extrativa. O resultado é compatível com a teoria da discriminação, indicando que pode existir discriminação de gênero nas capitais brasileiras nesses setores.

No modelo, foram atribuídos dummies para a escolaridade dos trabalhadores e o nível médio foi a variável omitida. Elas representam o capital humano e indicam a importância da educação para aumentar a renda dos trabalhadores. Os coeficientes da regressão para os trabalhadores que são analfabetos, que estudaram até completar o ensino fundamental e que não completaram o ensino médio, apresentaram sinal negativo, indicando que recebem remunerações menores que os trabalhadores que completaram o ensino médio. Os analfabetos ganham aproximadamente $60 \%$ a menos, os trabalhadores apenas com o ensino fundamental 3,5\% a menos e os trabalhadores com ensino médio incompleto $7,2 \%$ a menos que um trabalhador com ensino médio completo.

Os trabalhadores que possuem o ensino superior completo, incompleto e com pós-graduação apresentaram coeficientes com sinal positivo, demonstrando que recebem remuneração maior dos que os trabalhadores que estudaram apenas até o ensino médio. As magnitudes de acréscimo são $42,8 \%$ para trabalhadores com ensino superior incompleto, $371,7 \%$ para superior completo e $662,3 \%$ para pós-graduação. No modelo, a escolaridade dos trabalhadores se apresentou conforme a teoria 
do capital humano e indicou que quanto mais os trabalhadores investem tempo e dinheiro para sua especialização maior é o aumento de produtividade e, em consequência, aumento de renda.

Trabalhadores que concluem o ensino superior passam a ganhar 3,3 vezes mais do que os trabalhadores com o ensino superior incompleto. Os trabalhadores com pós-graduação ganham 1,6 vezes mais do que pessoas com apenas o ensino superior completo. Essas evidências mostram que os acréscimos nos salários dos trabalhadores são cada vez menores para níveis mais altos de escolaridade. Um trabalhador da agropecuária e indústria extrativa com pós graduação ganha ainda 19 vezes mais que um trabalhador analfabeto nesse mesmo setor.

\section{CONSIDERAÇÕES FINAIS}

Existe uma série de fatores apontados na literatura que podem levar à existência de diferenciais de produtividade entre os indivíduos e regiões e, portanto, afetar o nível da desigualdade de rendimentos. Dentre esses fatores estão os investimentos em capital humano, a qualidade do capital humano, as externalidades ou spillovers do conhecimento e das vantagens das aglomerações, as compensações em virtude de amenidades locais e os fatores advindos da discriminação.

O objetivo do trabalho foi estimar as implicações do estoque de capital humano sobre a produtividade individual dos trabalhadores, em consequência, para os rendimentos do setor agropecuário e da indústria extrativa das 26 capitais dos estados brasileiros e da capital do Distrito Federal. Além disso, verificar a influência de outras variáveis, como discriminação e diferenciais compensatórios, na definiçãa da produtividade individual. A fonte principal dos dados foi a RAIS no âmbito de indivíduo para o ano de 2015.

Os resultados mostraram que, ao investir em capital humano, os trabalhadores da agropecuária e da indústria extrativa aumentam seu nível de renda. Verificou-se a existência de rendimentos crescentes, mas com taxas marginais decrescentes para a escolaridade adicional (acréscimos nos salários cada vez menores para níveis mais altos de escolaridade), pois um trabalhador com ensino superior completo ganha 3,3 vezes mais do que os trabalhadores com superior incompleto e trabalhadores com pós-graduação ganham 1,6 vezes mais do que pessoas com apenas o ensino superior completo. Os resultados indicam ainda que um trabalhador com pós graduação ganha cerca de 19 vezes mais que um trabalhador analfabeto. Dessa forma, os resultados mostraram investimentos com retornos marginais crescentes de acordo com a idade e experiência. 
Como resultado, ainda, se verificou que a qualidade da educação contribui de forma positiva para os trabalhadores da agropecuária e indústria extrativa se tornarem mais produtivos, os homens auferem salários maiores que as mulheres e os trabalhadores não são compensados com salários maiores se as condições do trabalho e da localidade são ruins.

Algumas variáveis do modelo não apresentaram os sinais esperados, indicando uma limitação do trabalho. A falta de alguns dados para a escolha das proxies pode ter contribuído para isso. Não é objetivo do estudo encerrar as discussões sobre o tema. Sugere-se o estudo para outras localidades, outros setores, outros períodos para poder se realizar uma comparação e inclusão de outras variáveis. Além disso, se faz necessário, para dar maior robustez à análise, inclusão de dummies regionais e uma maior desagregar do setor agropecuário e extrativo.

Outro ponto importante a ser considerado para trabalhos futuros é a utilização de regressões quantílicas, que se possibilitarão verificar se as diferenças nos rendimentos se alteraram de acordo com os pontos ou grupos de distribuição salarial.

\section{REFERÊNCIAS}

ARRAES, R. de A.; MARIANO, F. Z. Endogeneidade da Educação na Previsão da Taxa de Retorno: Avaliação Metodológica e Aplicação para Regiões Brasileiras e Estados Selecionados. Rev. Econ. NE, v. 45, n. 2, p. 125-139, Fortaleza, 2014.

ARRAIS, S. C. da S.; PRAT, B. V.; CAMBRAIA, R.P. Análise dos Censos Agropecuários Brasileiros dos Anos de 2006 e 2017 para Identificação de Características da População Agrícola. Revista Cerrados, v. 17, n. 02, p. 228-246, 2019.

BRASIL. Ministério do Trabalho e do Emprego. RAIS- Relação Anual de Informações Sociais. 2020.

BECKER, G. S. Investment in human capital: A theoretical analysis. Journal of political economy, Chicago, v. 70, n. 5, p. 9-49, Oct. 1962.

BECKER, G. S. Human Capital Revisited. In: BECKER, G.S. Human capital: A theoretical and empirical analysis, with special reference to education. 3rd ed. Chicago: University of Chicago Press, Cap. 2, p. 15-28,1994.

CACCIAMALI, M. C.; HIRATA, G. I. A influência da raça e do gênero nas oportunidades de obtenção de renda-uma análise da discriminação em mercados de trabalho distintos: Bahia e São Paulo. Estudos Econômicos (São Paulo), v. 35, n. 4, p. 767-795, 2005. 
CARVALHO, A. P. de; NERI, M. C.; SILVA, D. B. Diferenciais de Salários por Raça e Gênero: Aplicação dos procedimentos de Oaxaca e Heckman em Pesquisas Amostrais Complexas. Ensaios Econômicos, Rio de Janeiro: FGV, n. 638, dez. 2006.

CEPEA- Centro de Estudos Avançados em Economia Aplicada. PIB do Agronegócio do Brasil de 1996 a 2018. 2018.

CHAVES, A. L. L. Estimativa da discriminação salarial, por gênero, para os trabalhadores assalariados da Região Metropolitana de Porto Alegre. Mulher e Trabalho, Porto Alegre, v. 2, p. 85-94, 2011.

CICCONE, A.; PERI, G. "Identifying human-capital externalities: Theory with applications". The Review of Economic Studies, v. 73, n. 2, p. 381-412, 2006.

CONFERÊNCIA NACIONAL DA INDÚSTRIA- CNI. Indústria em Números. Fev. 2020.

COSTA, C. C. de M.; ALMEIDA, A. L. T de.; FERREIRA, M. A. M.; SILVA, E. A. Determinantes do desenvolvimento do setor agropecuário nos municípios. Revista de Administração, v. 48, n. 2 , p. 295-309, 2013.

EHRENBERG, R. G.; SMITH, R. S. A moderna economia do trabalho: teoria e política pública. Tradução Sidney Stancatti,5. ed. São Paulo: Makron Books, 2000.

FÁVERO, L. P. L; BELFIORE, P. P.; SILVA, F. L. da; CHAN, B. L. Análise de dados: modelagem multivariada para tomada de decisões. Rio de Janeiro: Campus, 2009.

FERNANDES, R. Desigualdade salarial: aspectos teóricos. Estrutura salarial: aspectos conceituais e novos resultados para o Brasil. Rio de Janeiro: IPEA, p. 1-50, 2002.

FERNANDES, G. A. de A. L.. Brazilian female labor market: racial-skin color discrimination and inefficiency. Revista Economia Aplicada, São Paulo, v. 19, n. 2, p. 241-259, abr.jun. 2015.

FONTES, G. G.; SIMÕES, R. F.; OLIVEIRA, A. M. H. C. Diferenciais regionais de salário no Brasil, 1991 e 2000: uma aplicação dos modelos hierárquicos. Encontro Nacional de Economia, v. 34, 2006.

GALINARI, R.; COCO, M. A.; LEMOS, M. B.; BASQUES, M. F. D. O efeito das economias de aglomeração sobre os salários industriais: uma aplicação ao caso brasileiro. Revista Economia Contemporânea, v. 11, n. 3, p. 391-420, Rio de Janeiro, 2007.

GARCIA, J. R. Trabalho rural: tendências em face das transformações em curso. In: BUAINAIN, A. M.; ALVES, E.; SILVEIRA, J. M. da; NAVARRO, Z. (Ed.). O mundo rural no Brasil do século 21: a formação de um novo padrão agrário e agrícola. Brasília, DF: Embrapa, pg. 560-589, 2014.

GUJARATI, D. N; PORTER, D. C. Econometria Básica (Tradução da 5a edição Americana). Porto Alegre: AMGH, 2012. 
HANUSHEK, E. A; KIMKO, D. D. Schooling, labor-force quality, and the growth of nations. The American Economic Review, v. 90, n. 5, p. 1184-1208, 2000.

HEUERMANN, D. F. “Human capital externalities in Western Germany”. Institute for Labour Law and Industrial Relations in the European Community (IAAEG), University of Trier, 2009.

HEUERMANN, D.; HALFDANARSON, B.; SUEDEKUM, J. "Human capital externalities and the urban wage premium: Two literatures and their interrelations”. Urban Studies, v. 47, n. 4, p. 74, 2010 .

INEP - Instituto Nacional de Estudos e Pesquisas Educacionais Anísio Teixeira.

IBGE. Pesquisa Nacional por Amostra de Domicílios (PNAD) 2015.

LIMA, M. H. M. R. A indústria extrativa mineral: algumas questões socioeconômicas. In: BRASIL. Centro de Tecnologia Mineral. Tendências Tecnológicas Brasil 2015: Geociências e Tecnologia Mineral. Rio de Janeiro: CETEM/MCT, 2007. 372 p.

LOPES, L. M.; VASCONCELlOS, M. A. S. (Org.). Manual de Macroeconomia. São Paulo: Atlas, 3 ed. 2008.

MATTEI, T. F.; BAÇO, F. M. B. Análise das Desigualdades Salariais Entre Homens e Mulheres no Estado do Paraná. V Congresso Nacional de Pesquisa em Ciências Sociais Aplicadas, Anais Eletrônicos..., 2016.

MORETTI, E. "Estimating the social return to higher education: evidence from longitudinal and repeated cross-sectional data”. Journal of Econometrics, n.121, p.175-212, Jul.-Ago., 2004.

NUNES, P. A.; MORAES, M. L. de; ROSSONI, R. A. Eficiência da Agricultura Familiar nos Municípios Paranaenses. Revista Economia Ensaios, v. 34, n. 2, 2020.

RAMOS, L.; VIEIRA, M. L. Desigualdade de rendimentos no Brasil nas décadas de 80 e 90:evolução e principais determinantes. Texto para Discussão, Rio de Janeiro: IPEA, n. 803, jun. 2001.

RAIHER, A. P. Desenvolvimento Territorial: Uma Proposta Metodológica. In: BIDARRA, B.; FERRERA DE LIMA, J.; VOLL, F. P. (Org.). Economia e Desenvolvimento Territorial. Foz do Iguaçu: Editora Parque Itaipu, p. 36-46, 2017.

RIVADENEIRA, Luis. América Latina y el Caribe: crecimiento económico sostenido, población y desarrollo. CELADE, 2000.

ROCHA, R. C. B.; PERO, V. Discriminação racial e educação no Brasil. Sinais Sociais, Rio de Janeiro, v. 1, n. 33, p. 122-155, abr. 2007.

ROCHA, R. de M. et al. Externalidades do capital humano: Uma análise empírica para as cidades Brasileiras. In: Anais do XLI Encontro Nacional de Economia [Proceedings of the 41st Brazilian 
Economics Meeting]. ANPEC- Associação Nacional dos Centros de Pós-Graduação em Economia [Brazilian Association of Graduate Programs in Economics], 2014.

ROCHA, R. M.; NETO, R. da M. S.; GOMES, S. M. F. P. O. Maiores Cidades, Maiores Habilidades Produtivas: Ganhos de Aglomeração ou Atração de Habilidosos? Uma Análise para as Cidades Brasileiras. Revista Econômica do Nordeste, v. 42, n. 4, p. 675-696, 2016.

ROMER, P. M. Increasing returns and long-run growth. Journal of political economy, v. 94, n. 5, p. 1002-1037, 1986.

SANTOS, L. P. et al. Agronegócio Brasileiro no comércio internacional. Revista de Ciências Agrárias, v. 1, n. 39, p. 54-69, 2016.

SARAIVA, M. V. Dois ensaios sobre a qualidade regional do capital humano no Brasil. $82 \mathrm{f}$. Dissertação (Mestrado em Economia), Pontifícia Universidade Católica do Rio Grande do Sul, Porto Alegre, 2016.

SATEL, C. I. R. Desigualdade de rendimentos do trabalho no Paraná no período 2002 a 2009: uma análise quantílica para o quartil $0,25^{\circ}$ e percentil $0,90^{\circ}$ da distribuição de rendimentos. Dissertação (Mestrado em Economia Regional) -Universidade Estadual de Londrina- UEL, Centro de Estudos Sociais Aplicados, Londrina, 2011.

SCHULTZ, T. W. Investment in human capital. The American Economic Review, Pittsburgh, v. 51, n. 1, p. 1-17, Mar. 1961.

SCHUlTZ, T. W. Investindo no Povo. Tradução Élcio Gomes de Cerqueira. Rio de Janeiro: Forense Universitária, 1987.

SCHULTZ, T. W. O capital humano:Investimentos em educação e pesquisa. Tradução Marco Aurélio de Moura Matos. Rio de Janeiro: Zahar Editores, 1973.

SILVA, I. Teorias do emprego segundo o enfoque do capital humano, da segmentação e dos mercados internos. Revista da Fapese, Aracaju, v. 2, n. 2, p. 129-140, jul./dez. 2006

SILVA, N. de D. V.; KASSOUF, A. L. Mercados de trabalho formal e informal: Uma análise da discriminação e segmentação. Nova Economia, Belo Horizonte, v.10, n.1, julho de 2000.

TATEI, F. Desigualdades no mercado de trabalho da América Latina: a discriminação por sexo entre os trabalhadores com ensino superior no Brasil e no México. Dissertação (Mestrado em Integração da América Latina), Universidade de São Paulo- USP, Escola de Artes, Ciências e Humanidades, São Paulo, 2011.

VALLUIS, M. A. O Enade (Exame Nacional de Desempenho de Estudantes) Sob a Ótica de Alunos de Administração. 2014. 148f. Dissertação (Mestrado em Educação) - Programa de PósGraduação em Educação, Universidade Católica de Santos, Santos, 2014.

VIANA, G.; LIMA, J. F.de. La teoria del capital humano y el crecimiento económico. Interações (Campo Grande), v. 11, n. 2, p. 137-148, 2010. 